\title{
Effectiveness and Safety of Hypodermoclysis Patients With Cancer: A Single-Center Experience From Saudi Arabia
}

\author{
Sittelbenat Adem ${ }^{1}$, Nabil ALMouaalamy 2, 3, 4
}

1. Nursing, King Abdulaziz Medical City, Ministry of National Guard-Health Affairs, Jeddah, SAU 2. Oncology Department/Palliative, Princess Noorah Oncology Center, King Abdulaziz Medical City, Ministry of National GuardHealth Affairs, Jeddah, SAU 3. Research, King Abdullah International Medical Research Center, Jeddah, SAU 4. College of Medicine, King Saud Bin Abdulaziz University for Health Sciences, Jeddah, SAU

Corresponding author: Nabil ALMouaalamy, almoalami@yahoo.com

\begin{abstract}
Introduction: Decreased intake of food or fluid causes dehydration in hospitalized adult patients. This has led to a negative impact on patients and increased the morbidity and mortality rate at the Princess Noorah Oncology Center, where patients with advanced cancer who suffer from dehydration have been treated with parenteral fluids until the date of discharge from the hospital.
\end{abstract}

Objective: The objective of this pilot study is to assess the effectiveness and safety of hypodermoclysis (HDC) to close the gap of treatment for home-based palliative patients with cancer.

Method: During home visits, the home health care (HHC) nurse assessed these patients through history and physical examination for dehydration. Our team also incorporated the Edmonton Symptom Assessment System revised (ESAS-r) Scale in the assessment of these patients' symptoms. Informed consent has been obtained from the patient and the caregiver. The trained nurses initiated the subcutaneous infusion. The caregivers monitored it and disconnected it when completed. The study population consists of palliative patients with advanced cancer under the services of palliative care. Effectiveness and safety have been assessed using the ESAS-r scale.

Results: A total of 25 (92.6\%) HDC treatments were successfully completed for nine patients (seven males and two females). One female patient had only one session because her health had deteriorated for reasons other than dehydration and was transferred to the hospital. No serious side effects were observed. One (3.6\%) patient developed redness at the site of cannula insertion. The mean duration of the infusions was 8.44 hours. The median age was 70 years.

Conclusion: This study has concluded that HDC is effective, safe, and can enhance the patient's comfort level without the need for hospitalization. The fact that HDC can be administered at home with minimum equipment and technical support makes it an ideal option in several countries with varied income settings.

Review began 03/02/2021 Review ended 03/09/2021 Published 03/09/2021

\section{○ Copyright 2021}

Adem et al. This is an open access article distributed under the terms of the Creative Commons Attribution License CC-BY 4.0., which permits unrestricted use, distribution, and reproduction in any medium, provided the original author and source are credited.
Categories: Family/General Practice, Internal Medicine, Oncology

Keywords: hypodermoclysis, hydration, palliative care, dehydration, community, esas-r scale

\section{Introduction}

The World Health Organization (WHO) defines palliative care as an approach that concerns itself with the improvement of the quality of life of patients with life-threatening diseases. Palliative care aims to ameliorate patients' physical symptoms, such as pain, and relieve suffering. However, treating physical symptoms is not the only element of palliative care. Palliative care also provides psychosocial treatment by encouraging patients to live actively and not passively wait for death and spiritual support [1].

In advanced cancer patients when oral intake is insufficient to maintain adequate hydration, most patients in traditional hospitals receive parenteral fluids [2,3]. In contrast, advanced cancer patients receiving home hospice care almost never receive parenteral fluid [4,5]. The peripheral intravenous (IV) route for hydration may be problematic for advanced cancer patients and hold a potential problem in the home care setting $[6,7]$. Specifically, disadvantages of the IV route for hydration in such patients include pain associated with needle insertion, the need for frequent site changes, difficulty in finding venous access, the need for immobilization of the arm, impediments to mobility, the risk of increasing agitation, and accidental catheter removal in patients with delirium, the need for hospitalization, high cost, and need for specific training in surveillance and care of complications, such as thrombophlebitis and infection [8].

Hypodermoclysis (HDC) is a technique of infusing fluids slowly into the subcutaneous tissue to hydrate older patients with poor venous access. Compared to IV, HDC costs less and has minimal discomfort and 
complications [9]. The fluid is absorbed into circulation via diffusion and perfusion [10,11]. Hypodermoclysis has a low incidence of adverse effects, generally related to local effects, such as swelling, localized pain, and erythema [12]. Other potential advantages of hypodermoclysis are that it is easy to manage in the home care setting and that the tubing can be connected and disconnected from the needle by primary caregivers after minimal training, it does not require expensive and complex infusion pumps, and it has the capability of intermittent administration. Hypodermoclysis is suitable for use in many hospital and homecare situations regardless of the patient's age $[13,14]$.

Based on the available evidence, HDC can benefit patients with dehydration in conjunction with poor venous access who are unable to take adequate food or fluid $[15,16]$. With this in mind, our team felt that starting those patients on HDC with the help of caregivers (after training) may relieve those symptoms and help them achieve their goal of staying home with their loved ones as long as possible without jeopardizing their safety. In a hospital setting, at the Princess Noorah Oncology Center (PNOC), advanced cancer patients receive IV infusion for nutrition, medications, or hydration. Upon discharge, an appointment is set for a follow-up at the palliative clinic with referral to home health care (HHC). This pilot study has been conducted to assess the utility, safety, and effectiveness of HDC hydration on palliative patients with dehydration.

The added advantage of this project is to help patients stay at home. HDC can reduce emergency room visits and hospital re-admissions $[11,17]$. Caregivers in our Saudi society associate hydration with compassion and as a means of nurturing their loved ones.

\section{Materials And Methods}

The study follows the observation method to assess home-based cancer patients who had been discharged from PNOC between January 2015 and February 15, 2016. The patients were referred to HHC and were seen within 14 days from the discharge date. On discharge, the patients were comfortable and had fair oral intake and no signs of dehydration. Although our team knows that it's inevitable that those patients will get worse and will need more help later, we tried to achieve the patient and family wishes to be home with their loved ones. Before the initiation of the pilot project, all nurses were trained on how to start HDC and symptoms assessment before and after HDC. A 45-60 minute training session was given to all caregivers by the HHC palliative nurse on the administration of hypodermoclysis and infusion site assessment. In this study, we included adult patients with advanced cancer with dehydration (presented at home with decreased oral intake, lethargy, dry mouth, and scant urine output) in need of hydration and we excluded patients with hypovolemic shock and with no caregivers.

During the home visits, the HHC nurse assessed these patients through history and physical examination for dehydration, also our team have incorporated the Edmonton Symptom Assessment System revised (ESAS-r) Scale in the assessment of these patients' symptoms and also to assess the improvements through ESAS-r score as well following the HDC infusion. The assessment findings were passed to the palliative consultant who ordered the HDC infusion electronically and faxed it and documents it in the patient medical records. The fluid type was chosen based on the palliative physician's decision which was based on baseline sodium level at discharge and also patient comorbidities (e.g. diabetes, hypertension, and heart failure). The HDC technique and nursing training is shown in Appendix A. The appropriate sites for cannula insertion, such as the upper back, chest, outer arms, abdomen, and outer thighs [18] are the shaded areas in Figure 1 . The cannula was changed every 72-96 hours. The maximum volume delivered by gravity daily was $1000 \mathrm{ml}$. The rate of infusion was $0.5-2 \mathrm{ml}$ per minute. Fluids infused per site were $1000 \mathrm{ml}$ [19]. The duration of the infusion in a day was eight, 10 , or 12 hours for one liter and half the time for $500 \mathrm{ml}$. In this study, the infusion fluid has no additives. 


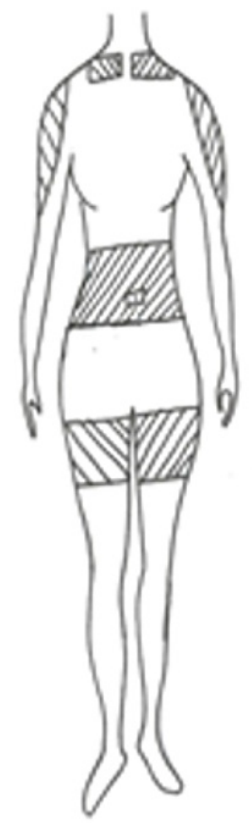

Front
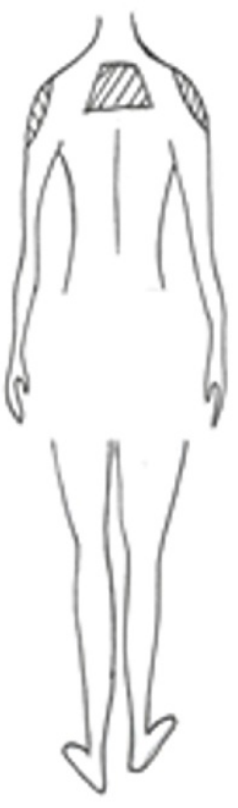

Back

\section{FIGURE 1: Site of Cannula Insertion (Shaded Areas)}

The HHC nurse obtained informed consent from them. The HHC nurse also calculated the rate based on the Manual of IV Therapeutics: Evidence-Based Practice for Infusion Therapy [20], prepared equipment, and initiated the infusion as in Appendix B.

The nurses start the infusion daily during the treatment period, and the family disconnects upon completion. The team focused on the identified issue using ESAS-r scale symptoms such as (pain, tiredness, nausea, depression, anxiety, drowsiness, appetite, feeling of well-being, and shortness of breath). The symptoms are categorized by severity: 0 (none), 1-3, (minimum) 4-6 (moderate), and 7-10 (severe) if the patient or family couldn't give a clear numerical value. The caregivers were contacted by phone every four hours. However, if they have any concerns, they were encouraged to contact the HHC nurse by phone.

The outcome of HDC was evaluated in terms of volume of fluid infused in 24 hours and improved dehydration symptoms that were present initially and also evaluated using the ESAS-r Scale before and after the treatment. The protocol used for applying HDC based on the hospital policy and procedures, Covenant Health Corporate Policy/Procedure \#VII-B-110, and Full Scope of Practice 2014 [13]. Data collection included: demographic data, functional status, duration of the infusion (three days), type of solution, indication, adverse effects, family response, and outcome.

\section{Results}

A total of 25 (92.6\%) HDC treatments were successfully completed in nine advanced cancer patients. The patients were treated and followed up at home under the palliative care department at PNOC in Jeddah, Saudi Arabia. Most of the patients (77.8\%) were males and the remainder were females. The mean age of the patients was equal to 71.89 years, $S D=10.82$ years, and most of them were married. Concerning their diagnosis, one patient had breast cancer, two patients had lung cancer, two had been diagnosed with colon cancer, one patient had gastric cancer, one patient had nasopharyngeal, another had hepatic, and one of them was diagnosed with prostate cancer. HDC treatments were successfully completed in nine patients (seven males and two females) (Table 1). 


\section{Cureus}

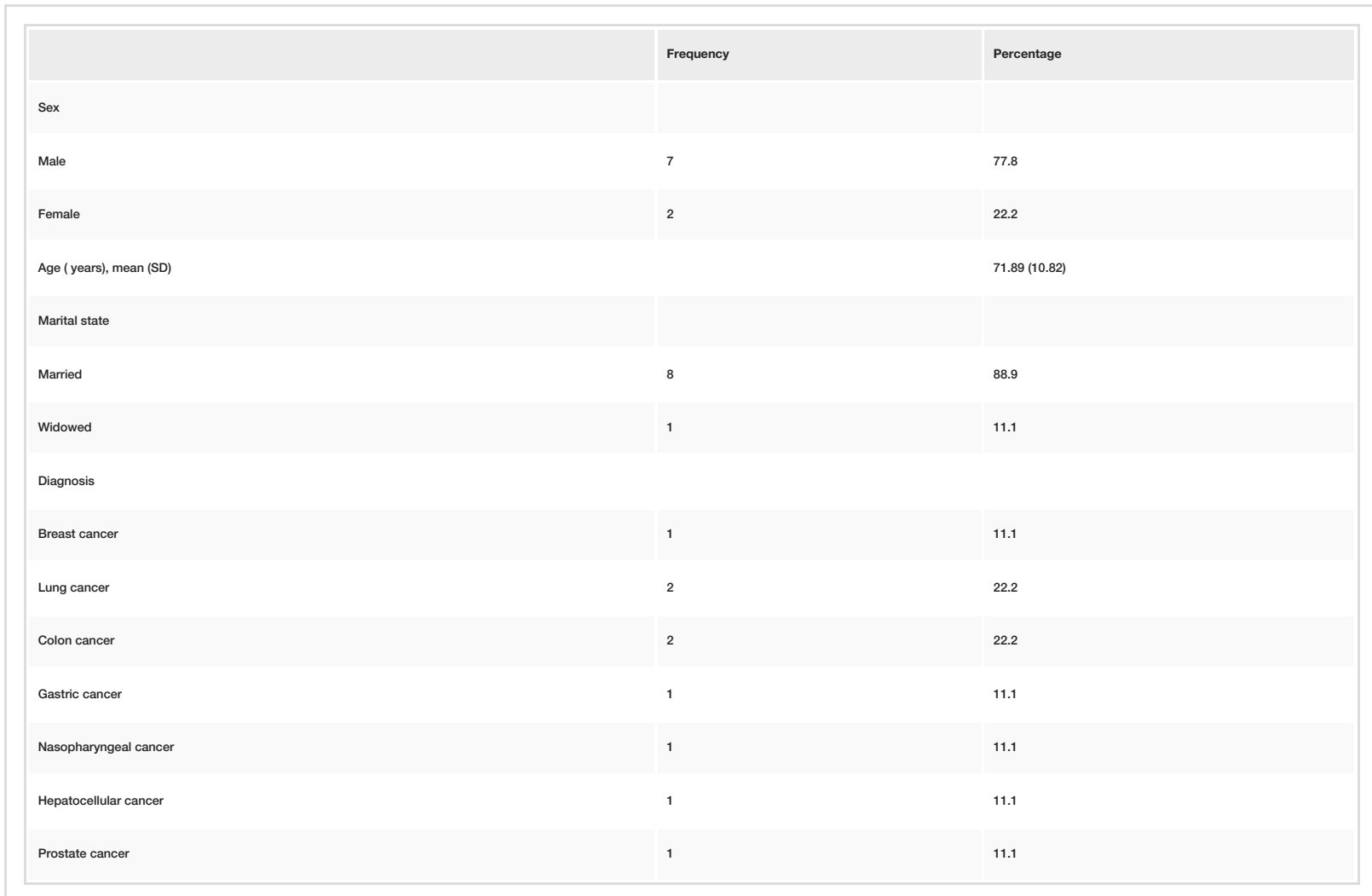

TABLE 1: Patients Sociodemographic and Disease-Related Characteristics. $\mathrm{N}=9$.

All the patients had required HDC therapy at home, $33.3 \%$ of the patients received sodium chloride $0.9 \%$ solution, another $22.2 \%$ had received a sodium chloride $0.45 \%$ solution, but $11.1 \%$ had required HDC infusion of dextrose $5 \%$ with sodium chloride $0.9 \%$ solution, but also $22.2 \%$ had required HDC infusion of dextrose $5 \%$ with sodium chloride $0.45 \%$ solution, and $11.1 \%$ required a dextrose $3.3 \%$ with sodium chloride $0.3 \%$ solution. The mean duration of infusion days was equal to 3.22 days, $S D=1.1$ days, and the mean duration of the infusion in hours was equal to 8.44 hours, $S D=2.74$ hours. However, most of the patients (66.7\%) had required a total of $1000 \mathrm{ml}$ of the infused hypodermal fluid solution and $33.3 \%$ of the patients required a total of $500 \mathrm{ml}$ of the hypodermal fluid solution. The family overall satisfaction with the therapy was measured, and most of the families were generally satisfied (Table 2). 


\section{Cureus}

\begin{tabular}{|c|c|c|}
\hline Type of Intravenous Fluid therapy used & Frequency & Percentage \\
\hline Sodium Chloride $0.9 \%$ & 3 & 33.3 \\
\hline Sodium Chloride 0.45\% & 2 & 22.2 \\
\hline Dextrose $5 \%$ \& Sodium Chloride $0.9 \%$ & 1 & 11.1 \\
\hline Dextrose $5 \%$ \& Sodium Chloride $0.45 \%$ & 2 & 22.2 \\
\hline Dextrose 3.3\% \& Sodium Chloride 0.3\% & 1 & 11.1 \\
\hline Days of HDC fluids & & $3.22(1.1)$ \\
\hline Hours of HDC fluids transfusion & & $8.44(2.74)$ \\
\hline \multicolumn{3}{|l|}{ Amounts of IV fluid administered } \\
\hline $500 \mathrm{ml}$ & 3 & ${ }^{33.3}$ \\
\hline $1000 \mathrm{ml}$ & 6 & 66.7 \\
\hline \multicolumn{3}{|l|}{ Patients' family overall satisfactio } \\
\hline Satisfied & 8 & 88.9 \\
\hline Undecided & 1 & 11.1 \\
\hline
\end{tabular}

TABLE 2: Descriptive analysis of the patients who received hypodermic fluid therapy.

However the main outcome was measured on the patients themselves across three days, as the patients were asked to rate themselves for the extent of all ESAS-r Scale symptoms using an 0-10 Likert-like scale with a greater rating denoting the greater extent of the symptom. The most statistically and clinically significant symptoms during the assessment were nausea, loss of energy, and loss of appetite (Table 3).

\begin{tabular}{|c|c|c|c|c|c|}
\hline & Day-1/time1 & Day-2/time2 & Day-3/time3 & test statistic & p-value \\
\hline Nausea [0-10 Likert-scale mean (SD) rating] & $5.56(1.01)$ & $4.00(1.8)$ & $2.67(1.32)$ & $x^{2}(2)=17$ & $<0.001$ \\
\hline Loss of appetite [0-10 Likert-scale mean (SD) rating] & $4.78(0.67)$ & $3.22(1.39)$ & $2.00(1.00)$ & $x^{2(2)}=17.5$ & $<0.001$ \\
\hline Loss of energy [0-10 Likert-scale mean (SD) rating] & $4.33(1.12)$ & $2.78(1.40)$ & $1.56(0.88)$ & $x^{2}(2)=15.10$ & 0.001 \\
\hline
\end{tabular}

TABLE 3: Descriptive analysis and non-parametric Friedman's test of the patients measured outcomes in response to hypodermic IVF therapy. N=9 Patients.

A Friedman's non-parametric test was used to assess the statistical significance of the mean difference on patients' self-rating for the three symptoms (nausea, loss of appetite, and loss of energy) across the three time points during receiving the hypodermic fluid replacement therapy days. The resulted descriptive analysis ( means and standard deviations ) of patients' perception of nausea, loss of appetite, and energy are displayed in Table 3. The Friedman's test showed that there has been a statistically significant decline in patients feelings of nausea across time during the hypodermic fluid replacement period, $\chi 2(2)=17, p<0.001$, it is evident that the mean perceived nausea had declined from day one to day two then at day three, suggesting a linear declining trend that could be associated with the fluid replacement, also the patients mean self-rated sense of loss of appetite had declined significantly from baseline to the second day then to the third time point while receiving the HDC, $\chi 2(2)=17.5$, $p<0.001$, according to the Friendman's nonparametric test of paired repeated measures, likewise the patients self-rated mean perceived sense of loss of energy had declined significantly from day one to day two and day three, the mean perceived loss of energy had declined substantially between day one and day three, $\chi 2(2)=15.10, p<0.001$ according to the Friedman's test (Figure 2). The post-hoc pairwise comparisons for the three outcomes across the three time points suggested that only the third time points measured nausea, loss of appetite and energy differed significantly from the baseline measures for those patients perceptions, $\mathrm{p}<0.001$ each respectively, the first and second day readings did not differ significantly for the three outcomes nonetheless. Furthermore, the patient's age did not correlate significantly with their perceptions of nausea, loss of appetite, and energy. However, the duration days had correlated significantly with the severity of nausea and loss of appetite and energy, 
$r>0.50, p<0.050$ each respectively well according to Pearson's test of correlation.

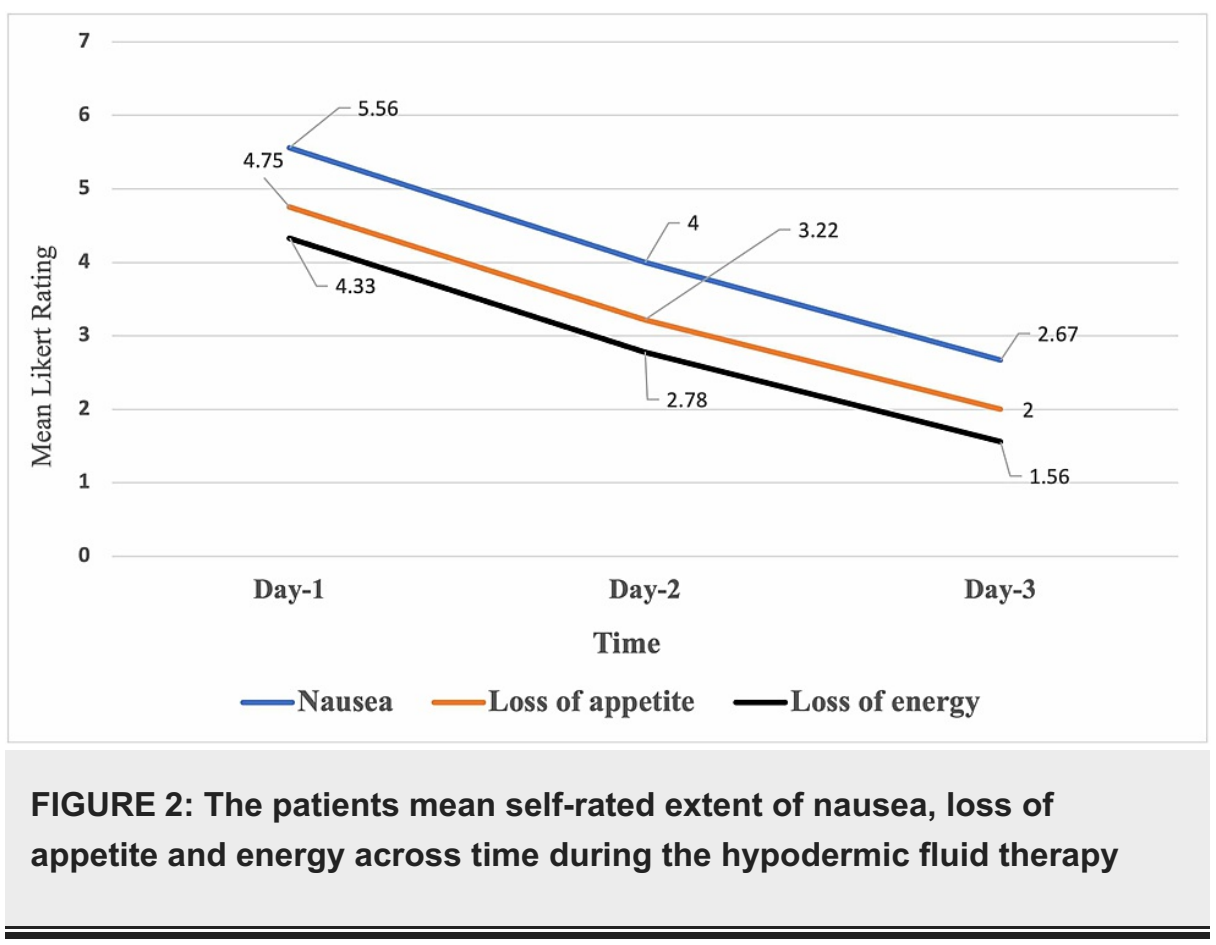

One patient (female) had one session but transferred to the hospital because her health deteriorated, not related to hydration. Regarding side effects, only one patient showed skin redness which subsided later with no intervention and was statistically insignificant.

\section{Discussion}

This pilot study suggests that hypodermoclysis hydration is an acceptable choice for advanced cancer patients in Saudi Arabia. In addition, it shows that minimal training to the caregiver can result in selfsatisfaction and help improving patient symptoms as well [21]. This study also shows that hypodermoclysis is a safe, effective, and useful hydration technique for palliative patients in our community $[22,23]$.

Our results show that the infusion of an average of $833 \mathrm{ml}$ was well tolerated over three to four days and made a significant impact on multiple symptoms, especially nausea, low energy, and low appetite, which was similar to what was found by Fainsinger et al. [5]. Our study also shows a hydration of an average of 833 $\mathrm{ml}$ over three to four days can help in hydrating advanced cancer patients and relieve some of their symptoms with minimal side effects and local toxicity, which correlates with the finding of a prospective open study of 100 consecutive patients in Canada, 69 of whom received subcutaneous hydration; the treatment was well tolerated in patients with an average volume of $1203 \pm 505 \mathrm{ml} /$ day although the infusion was continued for an average of $14 \pm 18$ days.

The duration of our HDC was three to four days due to logistic issues especially manpower. Our study is the first to show that hydration for three to four days can help and relieve some symptoms, Several studies have shown that hydration for four to seven days was beneficial and some took 14-18 days [24].

In this study, we attributed the insignificance change in pain score over the treatment period to the fact that our patients were just discharged from the hospital (within 14 days) and the pain was well controlled. Family satisfaction with the intervention was remarkable, mainly because most of our patients and families in our community would like to stay home as much as they can and this study show clearly that hypodermoclysis can help achieve that goal. The families felt safe and requested it. Our experience taught us that both patients and caregivers will consider HDC again in the future. Our team recommended that attending nurses be vigilant in assessing dehydration. In this study, our team delivered subcutaneous fluid at a rate of $41 \mathrm{ml}$ per hour. No pooling of fluid was noticed. The infusion time for $1000 \mathrm{ml}$ was eight, 10, or 12 hours at a single site (500 $\mathrm{ml}$ in half the time). Following the HDC guidelines eliminates the potential adverse effects of subcutaneous hydration. Local massage eliminates mild subcutaneous edema.

The duration of the infusion in a day was eight, 10, or 12 hours for $1 \mathrm{~L}$ and half the time for $500 \mathrm{ml}$. Since the solution runs slowly, no transient local edema was noticed. In this study, the infusion fluid has no additives.

Patient and family education and support promote safety and acceptance of subcutaneous hydration at home. This was emotionally beneficial for the patient and the caregiver. The Bioethics study showed an 
ethical dilemma when nutrition or fluid is withheld from palliative patients [25]. It is not surprising to see some families even demand fluid infusions at the end of life. However, the assessment has to be on an individual basis. In this study, the families expressed their satisfaction, showed the confidence to participate, and had a positive experience of the care. The families responded positively and requested the treatment, which continues for a few days more, as in Table 2.

We acknowledge that our study was limited because of a small sample of nine patients. There is a need for further studies in this area to validate outcomes with a larger sample.

\section{Limitations}

The sample size is small, which may decrease the statistical power, and is non-randomized. Furthermore, the published material on the topic is very limited. Only some observational or randomized controlled trial studies are available. Further study is needed, which will be designed as a randomized controlled trial to examine the efficacy of HDC infusion in providing drugs in a multicenter study.

\section{Conclusions}

In this pilot project, our team implemented hypodermoclysis with considerable success in the home setting. Based on our results, we concluded hypodermoclysis is a safe and effective way to provide clinically indicated fluid volumes in adult palliative patients with dehydration and is well accepted by patients and families in Saudi Arabia. Also, minimal training of caregivers in our community can help the patient and the family achieve their goal of keeping the patient home as much as possible safely. HDC may serve as a perfect way to increase patient and caregiver satisfaction, decrease ER visits, and increase hospital revenue.

\section{Appendices}

\section{Appendix A}

Training the nurses in-home health care

Training sessions for the nurses were prepared and delivered for a period of 60 minutes. The trainees practiced on a dummy first. They offered the infusion in the community under supervision.

Techniques of the Subcutaneous Infusion

Following the policy and procedure of the hospital and Covenant Health, we chose the proper sites for Cannula insertion. Choosing an insertion site depends on the available subcutaneous tissue and the state of the patient's condition as indicated.

Areas to avoid are broken skin or irradiated, little subcutaneous, areas with lymphedema, infection, and ascites.

Caregiver Training

The sessions for caregiver training ranged from 45 to 60 minutes. The home health care nurses hang the fluid, adjust the rate, and assess the site daily during the prescribed treatment period. The type of fluids ordered can be hypotonic, isotonic, or hypertonic, depending on the patient's condition.

Equipment

The choice of equipment is based on standard 64 of the Infusion Nurses Society's Infusion Nursing Standards of Practice.

Drip Rate Calculation

The following formula used to calculate drip rate:

Volume $\mathrm{x}$ Drop factor $=$ drops per minute.

Time in minutes

For instance, if the ordered fluid is $1000 \mathrm{ml}$ of $0.9 \%$ normal saline to be infused over 8 hours

$1000 \times 20=42$ drops per minute 


\section{Appendix B}

Initiation of Hypodermoclysis

1. Assemble the equipment (fluid and tubing).

2. Wash hands Don Gloves follow standard precautions and use aseptic technique.

The cannula insertion site is chosen based on adequate subcutaneous tissue, the patient's comfort, skin integrity, and patient's mobility.

3. Swab the site with chlorhexidine in a circular motion, beginning at the center of the site.

4. Insert the cannula with the needle, bevel up, in the subcutaneous tissue at a 45 to 60 -degree angle, toward the head of the patient.

5. Secure the needle and the tubing with an occlusive dressing (Tegaderm).

6. Adjust fluid drip rate as prescribed to be infused via gravity.

The choice of equipment is based on standard 64 of the Infusion Nurses Society's Infusion Nursing Standards of Practice.

Equipment

Gloves

1. $70 \%$ alcohol swab or chlorohexidine sticks

2. Standard fluid infusion extension set with the drip chamber4

3. Solution bag $(0.45 \%$ normal saline, $2 / 3$ dextrose $1 / 3$ normal saline infusion, $0.9 \%$ normal a saline and dextrose 5\%)

4. $70 \%$ alcohol swab or chlorohexidine sticks

5. Cannula 24 or 25 gauge

6. Semipermeable transparent dressing for insertion site e.g., Tegaderm

7. Drip stand (optional at home can use dress hanger) and

8. Sharps box

9. Sterile cap,

10. Gauze $2 \times 2 \mathrm{~cm}$

11. Sharps box.

\section{Additional Information}

\section{Disclosures}

Human subjects: Consent was obtained or waived by all participants in this study. King Abdullah International Medical Research Centre, Jeddah, Saudi Arabia. issued approval RJ20-169-J. After reviewing your proposal the IRB has approved your proposal. Animal subjects: All authors have confirmed that this study did not involve animal subjects or tissue. Conflicts of interest: In compliance with the ICMJE uniform disclosure form, all authors declare the following: Payment/services info: All authors have declared that no financial support was received from any organization for the submitted work. Financial relationships: All 
authors have declared that they have no financial relationships at present or within the previous three years with any organizations that might have an interest in the submitted work. Other relationships: All authors have declared that there are no other relationships or activities that could appear to have influenced the submitted work.

\section{Acknowledgements}

To all Home Health Care staff and nurses and the palliative care physicians and nurses at King Abdulaziz Medical City-Jeddah, National Guard Health Affairs.

\section{References}

1. WHO Definition of Palliative Care. (2012). http://www.who.int/cancer/palliative/definition/en/

2. MacDonald N, Fainsinger R: Indications and ethical considerations in the hydration of patients with advanced cancer. Cachexia-Anorexia in Cancer Patients. Bruera E, Higginson I (ed): Oxford University Press, New York; 1996. 1:94-109.

3. Bruera E, Belzile M, Watanabe S, Fainsinger RL: Volume of hydration in terminal cancer patients . Support Care Cancer. 1996, 4:147-150. 10.1007/BF01845764

4. Dunphy K, Finlay I, Rathbone G, Gilbert J, Hicks F: Rehydration in palliative and terminal care: if not -- why not?. Palliat Med. 1995, 9:221-228. 10.1177/026921639500900307

5. Fainsinger RL, Bruera E: Hypodermoclysis (HDC) for symptom control vs. the Edmonton injector (EI) . J Palliat Care. 1991, 7:5-8. 10.1177/082585979100700402

6. Steiner N, Bruera E: Methods of hydration in palliative care patients . J Palliat Care. 1998, 14:6-13. $10.1177 / 082585979801400202$

7. Dalal S, Bruera E: Dehydration in cancer patients: to treat or not to treat . J Support Oncol. 2004, 2:467-479.

8. Fainsinger RL: Dehydration and rehydration. Textbook of Palliative Medicine. Bruera E, Higginson I, Ripamonti C, von Gunten C (ed): Taylor \& Francis, London; 2006. 727-735.

9. Lopez JH, Reyes-Ortiz CA: Subcutaneous hydration by hypodermoclysis. Rev Clin Gerontol. 2010, 20:105113. 10.1017/S0959259810000109

10. Lybarger EH: Hypodermoclysis in the home and long-term care settings. J Infus Nurs. 2009, 32:40-43. 10.1097/NAN.0b013e3181922552

11. Berger EY: Nutrition by hypodermoclysis. J Am Geriatr Soc. 1984, 32:199-203. 10.1111/j.15325415.1984.tb02002.x

12. Frisoli JA, de Paula AP, Feldman D, Nasri F: Subcutaneous hydration by hypodermoclysis. A practical and low cost treatment for elderly patients. Drugs Aging. 2000, 16:313-319. 10.2165/00002512-20001604000007

13. Fainsinger RL, Bruera E: The management of dehydration in terminally ill patients . J Palliat Care. 1995, 10:55-59. 10.1177/082585979401000312

14. Schen RJ: Subcutaneous infusions in the elderly. Br Med J (Clin Res Ed). 1981, 282:823-824. 10.1136/bmj.282.6266.823-d

15. Caccialanza R, Constans, Cotogni $\mathrm{P}$, et al.: Subcutaneous infusion of fluids for hydration or nutrition: a review. J Parenter Enteral Nutr. 2018, 42:296-307. 10.1177/0148607116676593

16. Remington R, Hultman T: Hypodermoclysis to treat dehydration: a review of the evidence . J Am Geriatr Soc. 2007, 55:2051-2055. 10.1111/j.1532-5415.2007.01437.x

17. Junior AF, de Paula AP, Feldman D, Nasri F: Subcutaneous hydration by hypodermoclysis. A practical and low cost treatment for elderly patients. Drugs Aging. 2000, 16:313-9. 10.2165/00002512-200016040-00007

18. Slesak G, Schnürle JW, Kinzel E, Jakob J, Dietz K: Comparison of subcutaneous and intravenous rehydration in geriatric patients: a randomized trial. J Am Geriatr Soc. 2003, 51:155-160. 10.1046/j.15325415.2003.51052.x

19. Health Technology Inquiry Service: Volume and site preferences for hypodermoclysis; a review of clinical practice guidelines. CADTH. 2010, 1-17.

20. Hypodermoclysis (HDC) administration. (2017). http://extcontent.covenanthealth.ca/Policy/VII-B-315.pdf.

21. Saganski GF, de Souza Freire MH: Safety and effectiveness of hypodermoclysis compared to intravenous fluid infusion for rehydrating children with mild to moderate dehydration. JBI Database System Rev Implement Rep. 2019, 17:1270-1276. 10.11124/jbisrir-2017-003696

22. Duems-Noriega O, Arĩno-Blasco S: Subcutaneous fluid and drug delivery: safe, efficient, and inexpensive . Rev Clin Gerontol. 2015, 25:117-146. 10.1017/S095925981500012

23. Sasson M, Shvartzman P: Hypod: an alternative infusion technique . Am Fam Physician. 2001, 64:1575-1579. $10.1017 /$ S095925981500012X

24. Vidal M, Hui D, Williams J, Bruera E: A prospective study of hypodermoclysis performed by caregivers in the home setting. J Pain Symptom Manage. 2016, 52:570-574. 10.1016/j.jpainsymman.2016.04.009

25. Cohen MZ, Torres-Vigil I, Burbach BE, de Rosa, Bruera E: The meaning of parenteral hydration to family caregivers and patients with advanced cancer receiving hospice care. J Pain Symptom Manage. 2012, 43:855865. 10.1016/j.jpainsymman.2011.06.016 